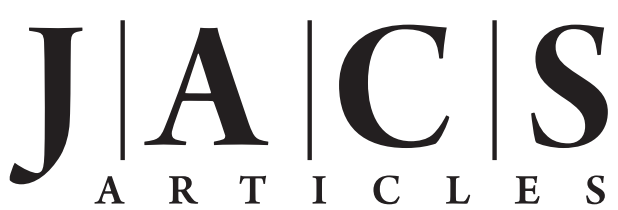

Published on Web 12/16/2010

\title{
Vibrational Relaxation and Intersystem Crossing of Binuclear Metal Complexes in Solution
}

\author{
Renske M. van der Veen, ${ }^{\dagger, \ddagger}$ Andrea Cannizzo, ${ }^{\dagger}$ Frank van Mourik, ${ }^{\dagger}$ Antonín Vlček, Jr., ${ }^{\S}$ \\ and Majed Chergui*, \\ Ecole Polytechnique Fédérale de Lausanne, Laboratory of Ultrafast Spectroscopy, ISIC, 1015 \\ Lausanne, Switzerland, Swiss Light Source, Paul Scherrer Institute, 5232 Villigen, Switzerland, \\ and School of Biological and Chemical Sciences, Queen Mary University of London, E1 4NS \\ London, United Kingdom
}

Received July 29, 2010; E-mail: majed.chergui@epfl.ch

\begin{abstract}
The ultrafast vibrational-electronic relaxation upon excitation into the singlet ${ }^{1} \mathrm{~A}_{2 \mathrm{u}}\left(\mathrm{d} \sigma^{*} \rightarrow \mathrm{p} \sigma\right)$ excited state of the $d^{8}-d^{8}$ binuclear complex $\left[\mathrm{Pt}_{2}\left(\mathrm{P}_{2} \mathrm{O}_{5} \mathrm{H}_{2}\right)_{4}\right]^{4-}$ has been investigated in different solvents by femtosecond polychromatic fluorescence up-conversion and femtosecond broadband transient absorption (TA) spectroscopy. Both sets of data exhibit clear signatures of vibrational relaxation and wave packet oscillations of the Pt-Pt stretch vibration in the ${ }^{1} \mathrm{~A}_{2 u}$ state with a period of $224 \mathrm{fs}$, that decay on a $1-2 \mathrm{ps}$ time scale, and of intersystem crossing (ISC) into the ${ }^{3} A_{2 u}$ state. The vibrational relaxation and ISC times exhibit a pronounced solvent dependence. We also extract from the TA measurements the spectral distribution of the wave packet at a given delay time, which reflects the distribution of $\mathrm{Pt}-\mathrm{Pt}$ bond distances as a function of time, i.e., the structural dynamics of the system. We clearly establish the vibrational relaxation and coherence decay processes, and we demonstrate that PtPOP represents a clear example of a harmonic oscillator that does not comply with the optical Bloch description due to very efficient coherence transfer between vibronic levels. We conclude that a direct $\mathrm{Pt}$-solvent energy dissipation channel accounts for the vibrational cooling in the singlet state. ISC from the ${ }^{1} \mathrm{~A}_{2 u}$ to the ${ }^{3} \mathrm{~A}_{2 u}$ state is induced by spin-vibronic coupling with a higher-lying triplet state and/or (transient) symmetry breaking in the ${ }^{1} \mathrm{~A}_{2 u}$ excited state. The particular structure, energetics, and symmetry of the molecule play a decisive role in determining the relatively slow rate of ISC, despite the large spin-orbit coupling strength of the $\mathrm{Pt}$ atoms.
\end{abstract}

\section{Introduction}

Binuclear $\mathrm{d}^{8}-\mathrm{d}^{8}$ molecular complexes of rhodium, iridium, and platinum bridged by various ligands have been extensively studied due to their interesting photochemical and photophysical properties arising from the lowest singlet and triplet ${ }^{1,3} \mathrm{~A}_{2 \mathrm{u}}$ $\left(\mathrm{d} \sigma^{* \rightarrow \mathrm{p} \sigma}\right)$ excited states. ${ }^{1-4}$ Based on the promotion of an electron from the antibonding $\mathrm{d} \sigma^{*}$ orbital $\left(\mathrm{d}_{z^{2}}\right.$-derived, $z$-axis along $\mathrm{Pt}-\mathrm{Pt}$ ) into the bonding $\mathrm{p} \sigma$ orbital $\left(\mathrm{p}_{z}\right.$-derived), the structural distortion characterizing the ${ }^{1,3} \mathrm{~A}_{2 u}$ states mainly involves a contraction of the metal-metal bond ${ }^{5-7}$ with a

† Ecole Polytechnique Fédérale de Lausanne.

Paul Scherrer Institute.

$\S$ Queen Mary University of London.

(1) Roundhill, D.; Gray, H.; Che, C. Acc. Chem. Res. 1989, 22, 55-61.

(2) Rice, S.; Milder, S.; Gray, H. Coord. Chem. Rev. 1982, 43, 349-354.

(3) Marshall, J.; Stiegman, A.; Gray, H. ACS Symp. Ser. 1986, 307, 166176.

(4) Miskowski, V.; Smith, T.; Loehr, T.; Gray, H. J. Am. Chem. Soc. 1985, 107, 7925-7934.

(5) Novozhilova, I.; Volkov, A.; Coppens, P. J. Am. Chem. Soc. 2003, $125,1079-1087$

(6) van der Veen, R. M.; Milne, C. J.; Nahhas, A. E.; Lima, F. A.; Pham, V.-T.; Best, J.; Weinstein, J. A.; Borca, C. N.; Abela, R.; Bressler, C.; Chergui, M. Angew. Chem., Int. Ed. 2009, 48, 2711-2714.

(7) Christensen, M.; Haldrup, K.; Bechgaard, K.; Feidenhans'1, R.; Kong, Q.; Cammarata, M.; Russo, M. L.; Wulff, M.; Harrit, N.; Nielsen, M. M. J. Am. Chem. Soc. 2009, 131, 502-508. concomitant increase of the symmetric metal-metal stretch vibrational frequency. ${ }^{8-10}$

Controlling the properties and behavior of these highly reactive excited states requires knowledge about the character and dynamics of the precursor states from which they are populated, ${ }^{11}$ but so far only a few studies have investigated excited-state decay processes in $\mathrm{d}^{8}-\mathrm{d}^{8}$ systems using steadystate $^{12}$ or time-resolved emission spectroscopy. ${ }^{13}$ Ultrafast spectroscopic methods are needed to unravel the various steps of the dynamics such as (coherent) vibrational dynamics, intramolecular energy redistribution, vibrational cooling, and intersystem crossing (ISC) and the way they are affected by the environment, as the latter can be used to tune the excitedstate properties.

Here we focus on the diplatinum(II) molecule $\left[\mathrm{Pt}_{2}-\right.$ $\left.\left(\mathrm{P}_{2} \mathrm{O}_{5} \mathrm{H}_{2}\right)_{4}\right]^{4-}$ (abbreviated PtPOP, see Figure 1), which is one of the most widely studied $\mathrm{d}^{8}-\mathrm{d}^{8}$ dimers owing to its unusual

(8) Fordyce, W.; Brummer, J.; Crosby, G. J. Am. Chem. Soc. 1981, 103, 7061-7064.

(9) Miskowski, V.; Rice, S.; Gray, H.; Dallinger, R. Inorg. Chem. 1994, 33, 2799-2807.

(10) Stiegman, A.; Rice, S.; Gray, H.; Miskowski, V. Inorg. Chem. 1987, 26, 1112-1116.

(11) Vlcek, A. Coord. Chem. Rev. 2000, 200, 933-977.

(12) Miskowski, V.; Rice, S.; Gray, H.; Milder, S. J. Phvs. Chem. 1993, 97, 4277-4283.

(13) Milder, S.; Brunschwig, B. J. Phys. Chem. 1992, 96, 2189-2196. 


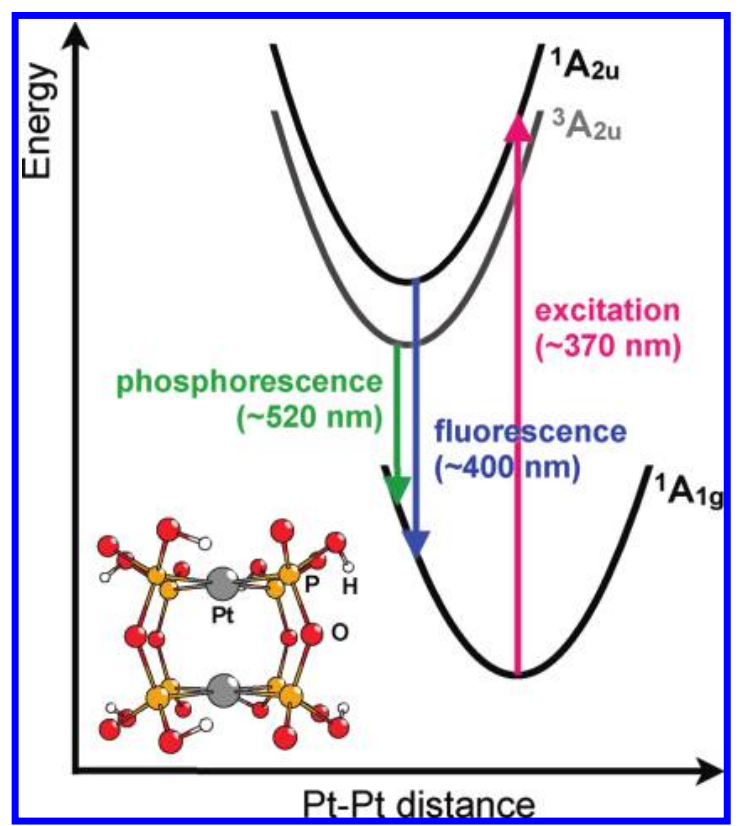

Figure 1. Simplified potential energy scheme of the ${ }^{1} \mathrm{~A}_{1 \mathrm{~g}}$ ground state and ${ }^{1,3} \mathrm{~A}_{2 \mathrm{u}}$ lowest-excited states of $\left[\mathrm{Pt}_{2}\left(\mathrm{P}_{2} \mathrm{O}_{5} \mathrm{H}_{2}\right)_{4}\right]^{4-}$ (PtPOP). Note that the ${ }^{1} \mathrm{~A}_{2 \mathrm{u}}$ and ${ }^{3} \mathrm{~A}_{2 \mathrm{u}}$ potential curves are vertically parallel. The optical transitions are indicated by vertical arrows. The molecular structure of PtPOP is shown on the left.

photochemical reactivity. ${ }^{1}$ The UV-visible absorption spectrum of PtPOP is characterized by a strong, narrow band around $370 \mathrm{~nm}$ due to absorption into the ${ }^{1} \mathrm{~A}_{2 \mathrm{u}}\left(\mathrm{d}-\sigma^{*} \rightarrow \mathrm{p} \sigma\right)$ state and a weak band around $460 \mathrm{~nm}$ assigned to the corresponding triplet ${ }^{3} \mathrm{~A}_{2 \mathrm{u}}$ state. Franck-Condon analyses of the low-temperature vibronic progressions of these absorption bands and their corresponding fluorescence and phosphorescence spectra (around 400 and $520 \mathrm{~nm}$, respectively, see Figure 1) have demonstrated that they are dominated by the $\mathrm{Pt}-\mathrm{Pt}$ stretch vibration, with a vibrational frequency of $\sim 150 \mathrm{~cm}^{-1}$ for the singlet and triplet absorption bands and $\sim 118 \mathrm{~cm}^{-1}$ for the emission bands. ${ }^{8-10,14}$

The mechanism of ISC, i.e., the nonradiative relaxation from the ${ }^{1} \mathrm{~A}_{2 \mathrm{u}}$ to the ${ }^{3} \mathrm{~A}_{2 \mathrm{u}}$ state, in binuclear $\mathrm{d}^{8}-\mathrm{d}^{8}$ complexes is still a subject of debate. ${ }^{15,13}$ In PtPOP, the formation of the triplet state from the singlet occurs with unity quantum yield ${ }^{8}$ on a picosecond time scale. ${ }^{10,13}$ Direct spin-orbit coupling between these states is, however, symmetry forbidden. In addition, their potential curves are vertically parallel ${ }^{10}$ (Figure 1) and separated by a large energy gap, while no intermediate state is known to lie between them. ${ }^{5,15}$ A higher-lying ${ }^{3} \mathrm{~B}_{2 u}$ triplet state has been invoked as a thermally activated channel for ISC in both $\mathrm{PtPOP}^{13}$ and $\mathrm{Rh}_{2} \mathrm{~b}_{4}{ }^{2+}(\mathrm{b}=\text { bridging ligand })^{12}$ complexes in order to explain the temperature dependence of the ISC rate.

In this paper we combine femtosecond polychromatic fluorescence up-conversion with femtosecond broadband transient absorption (TA) spectroscopy in order to elucidate the details of the early-time relaxation processes in the PtPOP anion, which have yet remained unresolved. This broadband experimental approach, in combination with the analysis of the transient spectra using singular value decomposition (SVD) and global fitting, allows us to unravel the details of the relaxation cascade during the first tens of picoseconds after excitation. We also

(14) Ikeyama, T.; Yamamoto, S.; Azumi, T. J. Phys. Chem. 1988, 92, 6899. (15) Shimizu, Y.; Tanaka, Y.; Azumi, T. J. Phvs. Chem. 1984, 88, $2423-$ 2425.

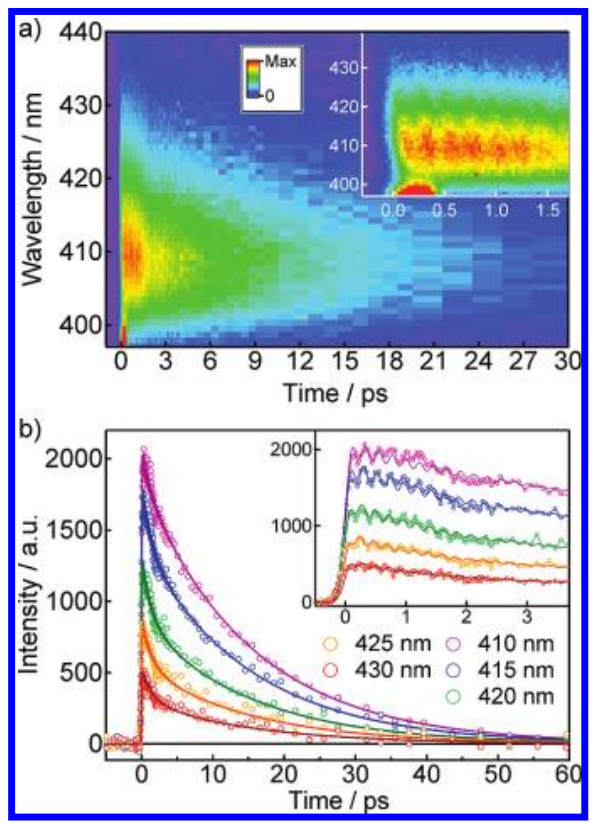

Figure 2. Time-resolved fluorescence data for PtPOP in water excited with a $\sim 120$ fs laser pulse at $380 \mathrm{~nm}$. (a) 2D time-wavelength plot of timeresolved fluorescence spectra. The inset shows a zoom into the initial 1.7 ps time window. (b) Fluorescence time traces (open circles) at various wavelengths (same data as in a), together with their fit functions using a global fit model (solid lines, see text). The inset shows a zoom into the initial 3.7 ps time window. The weak modulation during the first $3 \mathrm{ps}$ is due to a slow fluctuation in the laser power.

report on the observation of a vibrational wave packet evolving in the singlet-state potential. By means of spectrally and temporally resolving the stimulated emission, we fully map out the wave packet motion along the $\mathrm{Pt}-\mathrm{Pt}$ coordinate. Finally, we propose a simple relaxation model that could be applicable to the whole class of binuclear $\mathrm{d}^{8}-\mathrm{d}^{8}$ complexes, unifying the experimental observations of wave packet dynamics, vibrational relaxation, and ISC and their dependences on the excitation wavelength and the solvent. This work should also be cast in the context of our previous static and picosecond-resolved X-ray absorption studies of the geometric and electronic structures of the singlet ground ${ }^{16}$ and excited ${ }^{6,17}$ triplet states of the complex in solution. The present study addresses the electronic and structural relaxation dynamics at ultrashort time scales, which are so far not routinely accessible by X-ray techniques, and delivers insight into the important role of the environment in affecting them.

The experimental setup and details about the analysis are presented in the Supporting Information.

\section{Results and Analysis}

2.1. Femtosecond Polychromatic Fluorescence Up-Conversion. Figure 2 a shows a typical two-dimensional (2D) timewavelength plot of the time-resolved fluorescence spectrum obtained upon excitation of aqueous PtPOP at $380 \mathrm{~nm}$. Due to excessive scatter from the excitation beam, only the red part $>400 \mathrm{~nm}$ of the fluorescence band could be detected (see

(16) van der Veen, R.; Milne, C.; Pham, V.-T.; Nahhas, A. E.; Weinstein, J.; Best, J.; Borca, C.; Bressler, C.; Chergui, M. Chimia 2008, 62, 287-290.

(17) van der Veen, R. M.; Kas, J. J.; Milne, C. J.; Pham, V. T.; Nahhas, A. E.; Lima, F. A.; Amarasinge, D.; Rehr, J. J.; Abela, R.; Chergui, M. Phys. Chem. Chem. Phys. 2010, 12, 5551-5561. 
Table 1. Comparison of Relaxation Time Constants (Standard Deviations in Parentheses) for PtPOP in Four Different Solvents, Obtained from the Analysis of the Transient Absorption (TA) (Excitation at $370 \mathrm{~nm}$ ) and the Time-Resolved Fluorescence Up-Conversion (FU) Data (Excitation at $380 \mathrm{~nm})^{a}$

\begin{tabular}{|c|c|c|c|c|c|c|c|c|}
\hline \multirow[b]{2}{*}{ solvent } & \multirow{2}{*}{$\frac{\tau_{1} / \mathrm{fs}}{\mathrm{TA}}$} & \multicolumn{2}{|c|}{$\tau_{2} / \mathrm{ps}$} & \multicolumn{2}{|c|}{$\tau_{\mathrm{c}} / \mathrm{ps}$} & \multicolumn{2}{|c|}{$\tau_{3} / \mathrm{ps}$} & \multirow{2}{*}{$\begin{array}{c}T_{1} / \mathrm{fs} \\
\mathrm{TA}\end{array}$} \\
\hline & & $\mathrm{TA}$ & FU & TA & FU & TA & $\mathrm{FU}$ & \\
\hline ethylene glycol & $260(20)$ & $2.14(0.02)$ & & $1.93(0.04)$ & & $30.3(0.2)$ & & $224.0(0.1)$ \\
\hline ethanol & $280(70)$ & $2.11(0.03)$ & $2.0(0.2)$ & $2.30(0.08)$ & $2.3(0.4)$ & $25.6(0.2)$ & $28.9(0.4)$ & $223.8(0.1)$ \\
\hline DMF & $45(5)$ & $1.69(0.04)$ & & $1.75(0.08)$ & & $11.0(0.1)$ & & $222.8(0.1)$ \\
\hline
\end{tabular}

${ }^{a} \tau_{1}$ and $\tau_{2}$ are vibrational relaxation times, $\tau_{\mathrm{C}}$ is the coherence decay time, $\tau_{3}$ is the intersystem crossing time, and $T_{1}$ is the coherent oscillation period in the excited-state potential. The $\tau_{\mathrm{C}}$ times were obtained from a global analysis on a set of representative kinetic traces.

Supporting Information, section S1.1). Cuts at fixed emission wavelengths provide kinetic traces, shown in Figures $2 b$ and S1. The emission in the $400 \mathrm{~nm}$ region is due to fluorescence from the initially excited ${ }^{1} \mathrm{~A}_{2 \mathrm{u}}$ state. ${ }^{13}$ At least two kinetic components are contained in the data, and the emission shows clear modulations at early times (see insets) due to vibrational coherences (wave packets) in the ${ }^{1} \mathrm{~A}_{2 u}$ state potential. The traces of Figures $2 b$ and S1 were globally fitted to a sum of two exponential decay components convoluted with the experimental response function (190 fs fwhm) and an oscillatory component multiplied by an exponential decay representing the wave packet oscillation and coherence decay, respectively (see eq S5 in the Supporting Information). The best fit yields the kinetic time constants that are listed in Table 1. Short (1.5-2 ps) and long (15-30 ps) components show up, which are solvent dependent, as is also the damping time of the oscillations. The long decay component can be identified as the rate of ISC, while the short one, manifested as a narrowing of the emission band, is attributed to vibrational cooling in the singlet ${ }^{1} \mathrm{~A}_{2 \mathrm{u}}$ state. The oscillation period is $224 \pm 1 \mathrm{fs}\left(149 \mathrm{~cm}^{-1}\right.$, independent of the solvent), in good agreement with the excited ${ }^{1} \mathrm{~A}_{2 u}$ state $\mathrm{Pt}-\mathrm{Pt}$ vibration frequency from steady-state absorption spectroscopy. ${ }^{8,18}$ These results are complemented by detailed TA studies in a broader range of solvents.

\subsection{Femtosecond Broadband Transient Absorption Spec-} troscopy. Figure 3 a shows a representative set of timewavelength TA data upon excitation at $370 \mathrm{~nm}$ into the ${ }^{1} \mathrm{~A}_{2 \mathrm{u}}$ $\left(\mathrm{d} \sigma^{*} \rightarrow \mathrm{p} \sigma\right)$ band of PtPOP in ethylene glycol. All data have been corrected for group velocity dispersion as described in the Supporting Information (section S1.2).

The transient spectra contain four features: two excited-state absorptions (ESA, positive change in optical density, but plotted against the negative ordinate axis), the ground-state bleach (GSB, the dip at $370 \mathrm{~nm}$ is a pump artifact), and stimulated emission (SE, both negative change in optical density, but plotted against the positive ordinate axis). The latter two features overlap in the $370-400 \mathrm{~nm}$ region. The GSB remains unchanged up to the longest time delay of our experiment $(1.6 \mathrm{~ns})$, in agreement with the final excited state $\left({ }^{3} \mathrm{~A}_{2 u}\right)$ lifetime of several microseconds. ${ }^{8}$ Based on Figure 2a, the SE band around $400 \mathrm{~nm}$ is due to the ${ }^{1} \mathrm{~A}_{2 \mathrm{u}}$ state. The ESA bands around $335\left(\mathrm{ESA}_{1}\right)$ and $460 \mathrm{~nm}\left(\mathrm{ESA}_{2}\right)$ are present at all time delays but seem to red-shift on the time scale of several to tens of picoseconds. The evolution is more pronounced for $\mathrm{ESA}_{2}$ than $\mathrm{ESA}_{1}$. Their large intensity indicates that they belong to allowed transitions from the ${ }^{1} \mathrm{~A}_{2 \mathrm{u}}$ (at early times) and ${ }^{3} \mathrm{~A}_{2 \mathrm{u}}$ (at late times) excited states (vide infra). Figure $3 \mathrm{~b}$ shows a $3 \mathrm{D}$ zoom into the region of the SE band. It reveals a spectral substructure that initially decays in the very red wing while increasing closer to

(18) Rice, S.; Gray, H. J. Am. Chem. Soc. 1983, 105, 4571-4575.

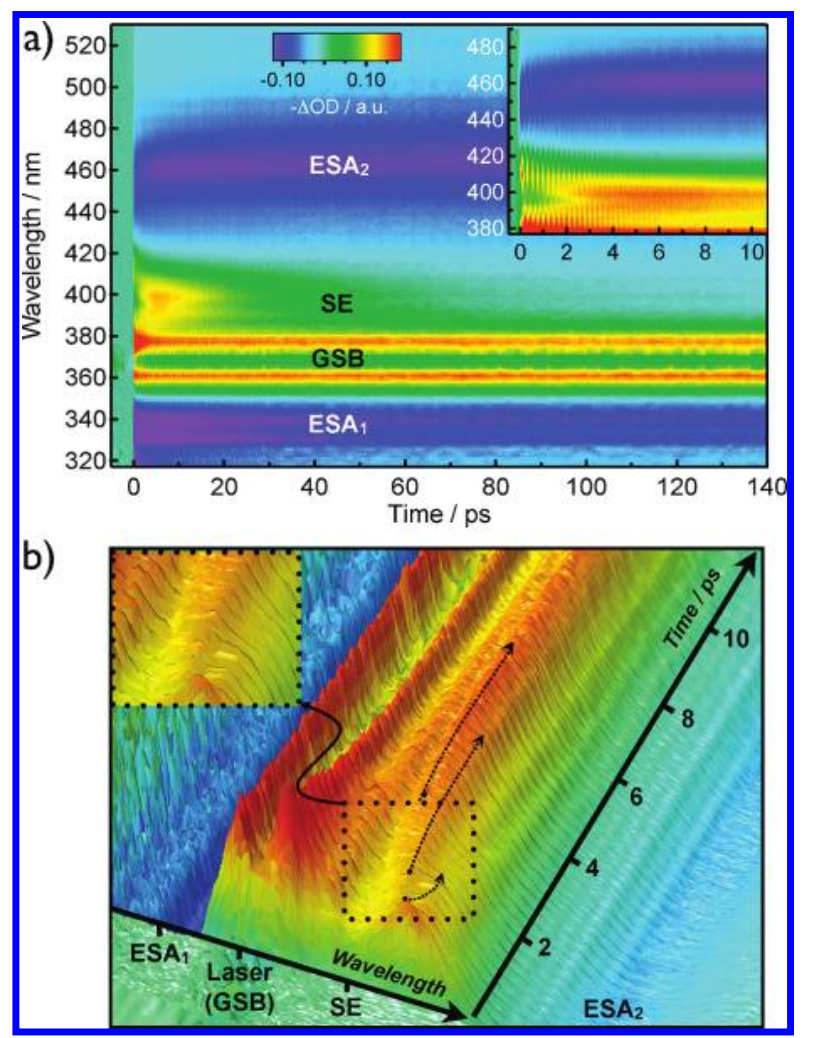

Figure 3. Transient absorption (TA) spectra for PtPOP in ethylene glycol excited with a $\sim 100 \mathrm{fs}$ laser pulse at $370 \mathrm{~nm}$. (a) $2 \mathrm{D}$ time-wavelength plot of the TA data. The inset zooms into the initial $10 \mathrm{ps}$. (b) 3D time-wavelength $-\triangle \mathrm{OD}$ plot zoomed into the region of the SE band which partially overlaps with the GSB in the region $<400 \mathrm{~nm}$ (same data as in a). One recognizes periodic intensity modulations and a spectral fine structure in the long-wavelength lobe of the SE band, indicated by dashed arrows and magnified in the upper-left inset. The "hole" in the GSB band is a pump artifact. ESA, excited-state absorption; GSB, ground-state bleach; SE, stimulated emission.

the center of the band around $400 \mathrm{~nm}$. The central "hole" at early times is filled up on a time scale of $\sim 2$ ps.

Cuts at fixed time delays deliver the corresponding transient spectra, which are shown in Figure 4a. The ESA around $335 \mathrm{~nm}$ could be assigned to the $\mathrm{A}_{2 \mathrm{u}} \rightarrow \mathrm{A}_{1 \mathrm{~g}}\left(5 \mathrm{~d} \sigma \rightarrow 5 \mathrm{~d} \sigma^{*}\right)$ transition on the basis of the calculated $5 \mathrm{~d} \sigma-5 \mathrm{~d} \sigma^{*}$ splitting of $\sim 4 \mathrm{eV} .{ }^{5,17}$ The ESA around $460 \mathrm{~nm}$ most likely belongs to the transitions from the $A_{2 u}$ singlet and triplet states to ${ }^{1,3} E_{g}$ states that can be described by the electronic configuration $\left(5 \mathrm{~d}_{x z}\right.$, $\left.5 \mathrm{~d}_{y z}\right)^{7}(6 \mathrm{p} \sigma)^{1}$ (the $\mathrm{d}_{x z}, \mathrm{~d}_{y z}$ manifold splits into $\pi\left(\mathrm{e}_{\mathrm{u}}\right)$ and $\pi^{*}\left(\mathrm{e}_{\mathrm{g}}\right)$ levels for $\mathrm{a} \mathrm{d}^{8}-\mathrm{d}^{8}$ complex $\left.{ }^{10}\right)$. Additional information is contained in the kinetic traces, which for three characteristic wavelengths $(340,410$, and $460 \mathrm{~nm})$ are shown in Figure 4b. They reveal dynamics on the time scales of hundreds of femtoseconds (modulations), a few picoseconds, and tens of 


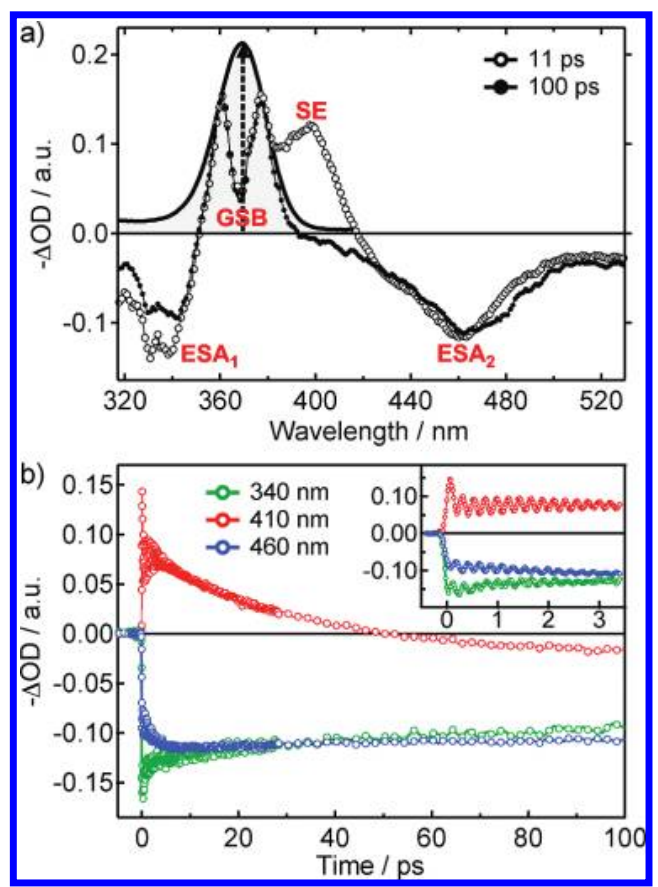

Figure 4. (a) Representative TA spectra at 11 (open circles) and $100 \mathrm{ps}$ (closed circles) after excitation at $370 \mathrm{~nm}$ into the ${ }^{1} \mathrm{~A}_{2 u}$ absorption band (dashed vertical arrow), together with a static absorption spectrum (black curve). The dip around $370 \mathrm{~nm}$ in the TA spectra is a pump artifact. (b) Time traces at fixed wavelengths. The upper-right inset zooms into the initial $3.5 \mathrm{ps}$ time window. $\triangle \mathrm{OD}$ is the change in optical density (pumped unpumped).

picoseconds, which are, based on the above fluorescence upconversion results, related to wave packet dynamics, vibrational cooling (in the ${ }^{1} \mathrm{~A}_{2 u}$ state), and ISC from the ${ }^{1} \mathrm{~A}_{2 u}$ state to the ${ }^{3} \mathrm{~A}_{2 \mathrm{u}}$ state, respectively.

The detailed analysis presented in the following sections allows us to quantify the time scales of these processes for various excitation wavelengths. In order to gain further insight into the mechanism of the relaxation processes, we measured TA spectra for samples of PtPOP dissolved in water, ethanol, and dimethylformamide (DMF). In addition, we will analyze the evolving spectral fine structure of the SE band seen in Figure $3 \mathrm{~b}$, which is identified as resulting from vibrational cooling and quantum mechanical interference in the vibrational coherent superposition state.

2.3. Spectro-Temporal Decomposition. In order to quantify and assign the kinetic and spectral components, we performed a singular value decomposition (SVD) analysis on the 2D data matrix (discussed in detail in the Supporting Information). The resulting kinetic amplitude vectors were globally fitted to a twostate kinetic model involving the lowest singlet and triplet $\mathrm{A}_{2 \mathrm{u}}$ states only, according to

$$
\begin{aligned}
f_{\text {kin }}(t) & =A_{1} \operatorname{ExpErf}\left[B, t_{0}, \tau_{1}, t\right] \cdots \\
& +A_{2} \operatorname{ExpErf}\left[B, t_{0}, \tau_{2}, t\right] \cdots \\
& +A_{3} \operatorname{ExpErf}\left[B, t_{0}, \tau_{3}, t\right] \cdots \\
& +A_{4}\left(\operatorname{ExpErf}\left[B, t_{0}, \tau_{4}=10 \mu \mathrm{s}, t\right]-\operatorname{ExpErf}\left[B, t_{0}, \tau_{3}, t\right]\right)
\end{aligned}
$$

where $\operatorname{Exp} \operatorname{Erf}\left[B, t_{0}, \tau, t\right]$ results from the convolution of an exponential function with a Gaussian function representing the instrumental response function (IRF) (see eq S3 in the Supporting Information for the mathematical definition), $A_{i}$ is the amplitude of the $i$ th one-exponential decay with time constant

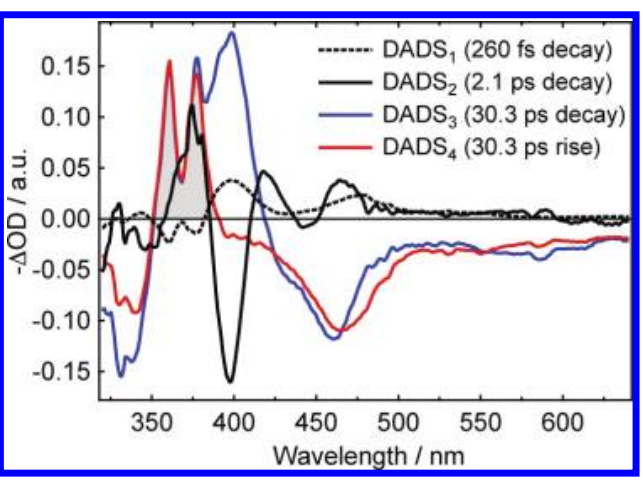

Figure 5. Decay-associated difference spectra (DADS) extracted from the TA data after SVD/global fit analysis using eq 1 . The gray area indicates the GSB region.

$\tau_{i}, B$ is the full width at half-maximum (fwhm) of the Gaussian IRF, and $t_{0}$ describes the time origin of the IRF (time zero). In the fits, the parameter $B$ was fixed to 0.13 ps (see Supporting Information).

Two independent exponential decay components (time constants $\tau_{1}$ and $\tau_{2}$ ) describe the vibrational dynamics within the initially excited ${ }^{1} \mathrm{~A}_{2 u}$ state, while the relaxation from the ${ }^{1} \mathrm{~A}_{2 u}$ to the ${ }^{3} \mathrm{~A}_{2 \mathrm{u}}$ excited state (i.e., ISC) is modeled by an exponential decay and rise with a single time constant $\left(\tau_{3}\right)$ but with different amplitudes ( $A_{3}$ and $A_{4}$, respectively). The $A_{4}$ term in eq 1 represents the arrival in the ${ }^{3} \mathrm{~A}_{2 \mathrm{u}}$ state and its subsequent decay with a time constant of $\tau_{4}=10 \mu$ s (not detectable on the maximum time scale of the experiment). The GSB feature in the spectrum is not explicitly included in the present model, but it appears naturally from the SVD as a spectral region with identical decay and rise amplitudes (see Figure 5).

The fit of the above-described model to the three largest kinetic amplitude vectors (see Supporting Information) delivered three time constants for PtPOP in ethylene glycol that are given in Table 1. The 30.3 ps decay is in good agreement with the long decay component in the fluorescence up-conversion data belonging to ISC from the ${ }^{1} \mathrm{~A}_{2 u}$ state to the ${ }^{3} \mathrm{~A}_{2 \mathrm{u}}$ state, while the fast 260 fs and 2.1 ps ( 2 ps in fluorescence up-conversion) decays represent vibrational dynamics and relaxation in the singlet state. ${ }^{19}$ Figure 5 shows the decay-associated difference spectra (DADS) reflecting the relative spectral contributions of each time component (see eq S4 in the Supporting Information). $\mathrm{DADS}_{3}$, associated with the departure from the ${ }^{1} \mathrm{~A}_{2 \mathrm{u}}$ state, exhibits the characteristic SE band around $400 \mathrm{~nm}$, which is absent in the DADS 4 spectrum belonging to the ${ }^{3} \mathrm{~A}_{2 \mathrm{u}}$ state. The ESA centered at $460 \mathrm{~nm}\left(\mathrm{ESA}_{2}\right)$ is slightly shifted and broadened in the ${ }^{3} \mathrm{~A}_{2 u}$ state compared to the ${ }^{1} \mathrm{~A}_{2 u}$ state, but overall the ESA spectra of the two states look very similar. $\mathrm{DADS}_{2}$ reflects the vibrational cooling in the ${ }^{1} \mathrm{~A}_{2 \mathrm{u}}$ state. Its decay with a time constant of 2.1 ps causes a narrowing of the singlet state SE band (vide infra, also seen in Figure 2a) and a red shift of the $\mathrm{ESA}_{2}$ absorption. The amplitude of the $\tau_{1}$ component is small, but its inclusion in the kinetic model has been found to considerably improve the fit of the short-time dynamics in the SVD kinetic amplitude vectors. This component is longer than the cross-phase modulation artifact around time zero (see Supporting Information for details).

(19) It is noted that it was not necessary to include the fast component in the fit model of the fluorescence up-conversion data. However, due to the limited data quality and the small spectral range of detection, the observation of this fluorescent component could well be obscured. 


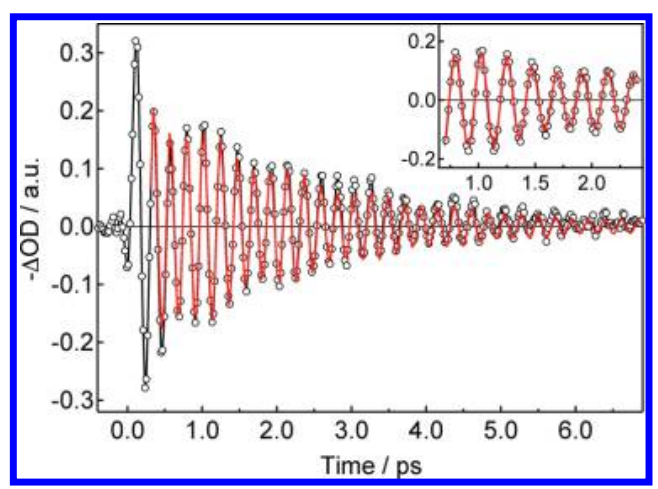

Figure 6. Linear combination of the fourth and fifth largest kinetic amplitude vectors from the SVD (open black circles), together with the fit to an exponentially damped superposition of two cosine functions with different periods (red curve). Fit results: $T_{1}=224 \mathrm{fs}, T_{2}=281 \mathrm{fs}$, and $\tau_{\mathrm{C}}$ $=2.1 \mathrm{ps}$. The inset shows a zoom into a short time range.

After having quantified the kinetic decay components, we now turn to the analysis of the vibrational coherences. Figure 6 shows the linear combination of the fourth and fifth largest time profiles from the SVD analysis scaled by their respective singular values (see Figure S2, Supporting Information). These vectors contain most of the oscillatory features in the TA spectrum and can therefore be used to determine the characteristic time constants of the wave packet dynamics, independent of the kinetic processes analyzed above. The coherent modulations strikingly persist for more than 5.5 ps after excitation $(\sim 25$ oscillations). The oscillatory trace of Figure 6 was fitted to a superposition of two cosine functions with different amplitudes $\left(A_{1}, A_{2}\right)$, phases $\left(\phi_{1}, \phi_{2}\right)$, and periods $\left(T_{1}, T_{2}\right)$, multiplied by an exponential decay function with time constant $\tau_{\mathrm{C}}$ being a quantitative measure for the coherence decay time of the wave packet dynamics, according to

$$
f_{\text {osc }}(t)=\mathrm{e}^{\left(t-t_{0}\right) / \tau_{C}}\left(A_{1} \cos \left[\frac{2 \pi t}{T_{1}}+\phi_{1}\right]+A_{2} \cos \left[\frac{2 \pi t}{T_{2}}+\phi_{2}\right]\right)
$$

An excellent fit is achieved for $T_{1}=224.0 \pm 0.1 \mathrm{fs}, T_{2}=281$ $\pm 1 \mathrm{fs}$, and $\tau_{\mathrm{C}}=2.16 \pm 0.05 \mathrm{ps}$ (the fit region started around $300 \mathrm{fs}$ to avoid the coherent artifact around time zero). The $T_{1}=224 \mathrm{fs}$ component is identified as the Pt-Pt stretch vibration in the ${ }^{1} \mathrm{~A}_{2 \mathrm{u}}$ excited state and is in very good agreement with the fluorescence up-conversion data. The superimposed 280 fs oscillation, causing the beat pattern in Figure 6, is due to coherent vibrational $\mathrm{Pt}-\mathrm{Pt}$ stretch oscillations in the groundstate (GS) potential that are excited via a resonant impulsive Raman-type process (resonant impulsive stimulated Raman scattering, RISRS). ${ }^{20}$ It is noted that the excited-state coherent oscillations are exceptionally harmonic and the coherence decay can be perfectly fit by a purely exponential decay function.

We also performed a global analysis of the TA spectra without prior decomposition of the data. A representative set of kinetic traces at fixed wavelengths were globally fitted to a function which is the sum of the population kinetics (eq 1) and the wave packet oscillations (eq 2, without the second cosine because its contribution was too weak) as employed in the SVD analysis. The global analysis result presented in Figure 7 shows a good match with the experimental traces over the whole spectrotemporal range, and the fit parameter values (see the Supporting

(20) Pollard, W.; Lee, S.; Mathies, R. J. Chem. Phvs. 1990, 92, 4012.

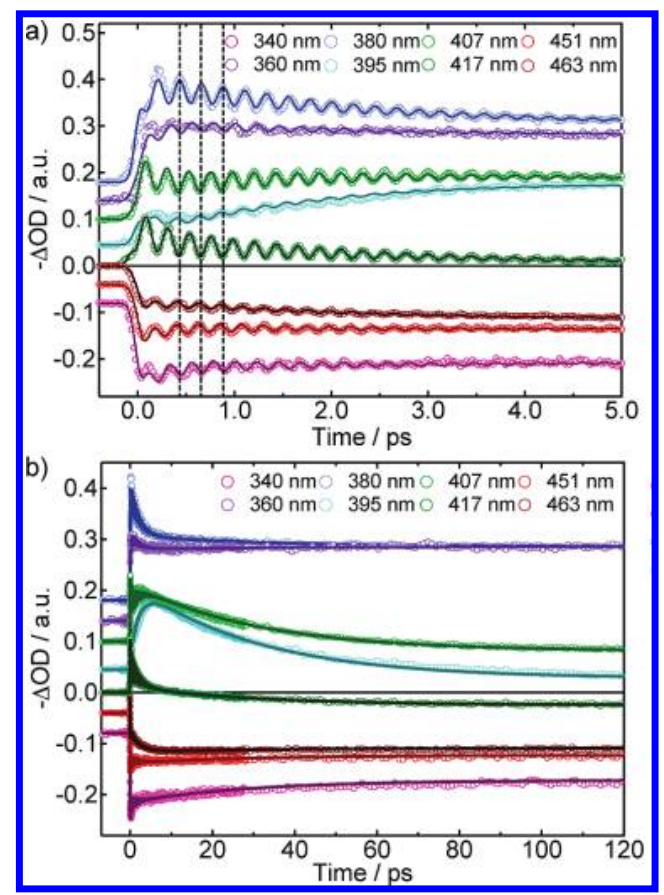

Figure 7. Kinetic traces from the transient absorption at fixed wavelengths, together with their fit functions using a global fit model (see text and eq S6 in the Supporting Information) for $2 \mathrm{mM}$ PtPOP in ethylene glycol excited at $370 \mathrm{~nm}$. (a) Short time range. The vertical dashed lines facilitate the observation of the phase shift of $\pi$ between the oscillations at the red and blue sides of the stimulated emission band (e.g., 407 and $380 \mathrm{~nm}$ ). (b) Long time range. The traces have been offset vertically for clarity.

Information for details) are in good agreement with the parameters from the SVD analysis. Characteristic of a wave packet oscillating between the classical turning point of the potential, the modulations exhibit a phase shift of approximately $\pi$ between the red and blue sides of the stimulated emission band, as is clearly visible for the traces at 407 and $380 \mathrm{~nm}$ in Figure $7 \mathrm{a}$.

Transient absorption data sets were measured for different excitation wavelengths $(360,370$, and $380 \mathrm{~nm})$ within the ${ }^{1} \mathrm{~A}_{2 u^{-}}$ state absorption band. SVD/GF and global analyses were performed on them in order to extract and compare the quantitative kinetic components (see Supporting Information, Tables S1, S2 and Figures S4, S5, for details). Time constant $\tau_{3}$, assigned to the ISC rate, was found to be independent of the excitation wavelength (within the measurement uncertainty). The rate of vibrational cooling $\left(\tau_{2}\right)$, however, changes by almost $40 \%$ for excitation between 360 and $380 \mathrm{~nm}$ (Table S1). The $\tau_{1}$ time constant could not be determined with sufficient accuracy for the off-resonance excitation wavelengths. For the sake of comparison, it was fixed to $0.2 \mathrm{ps}$. A very small lengthening of the wave packet period on the order of $1 \mathrm{fs}$ (or, equivalently, $\sim 1 \mathrm{~cm}^{-1}$ ) with increasing excitation energy is obtained from the analysis (see Table S2), which can be understood as resulting from a slight anharmonicity of the potential toward longer $\mathrm{Pt}-\mathrm{Pt}$ bond lengths, showing that the ${ }^{1} \mathrm{~A}_{2 \mathrm{u}}$ state potential is largely harmonic.

2.4. Solvent Effect on the Relaxation Processes. The results from the SVD/GF analysis of the TA data sets in the different solvents for excitation at $370 \mathrm{~nm}$ are given in Table 1 (see Supporting Information, Figures S8 and S9). In general, the TA and FU fitting results are in satisfactory agreement, given the different excitation wavelengths in the two experiments. Both the vibrational cooling $\left(\tau_{2}\right)$ and the ISC $\left(\tau_{3}\right)$ times show a 

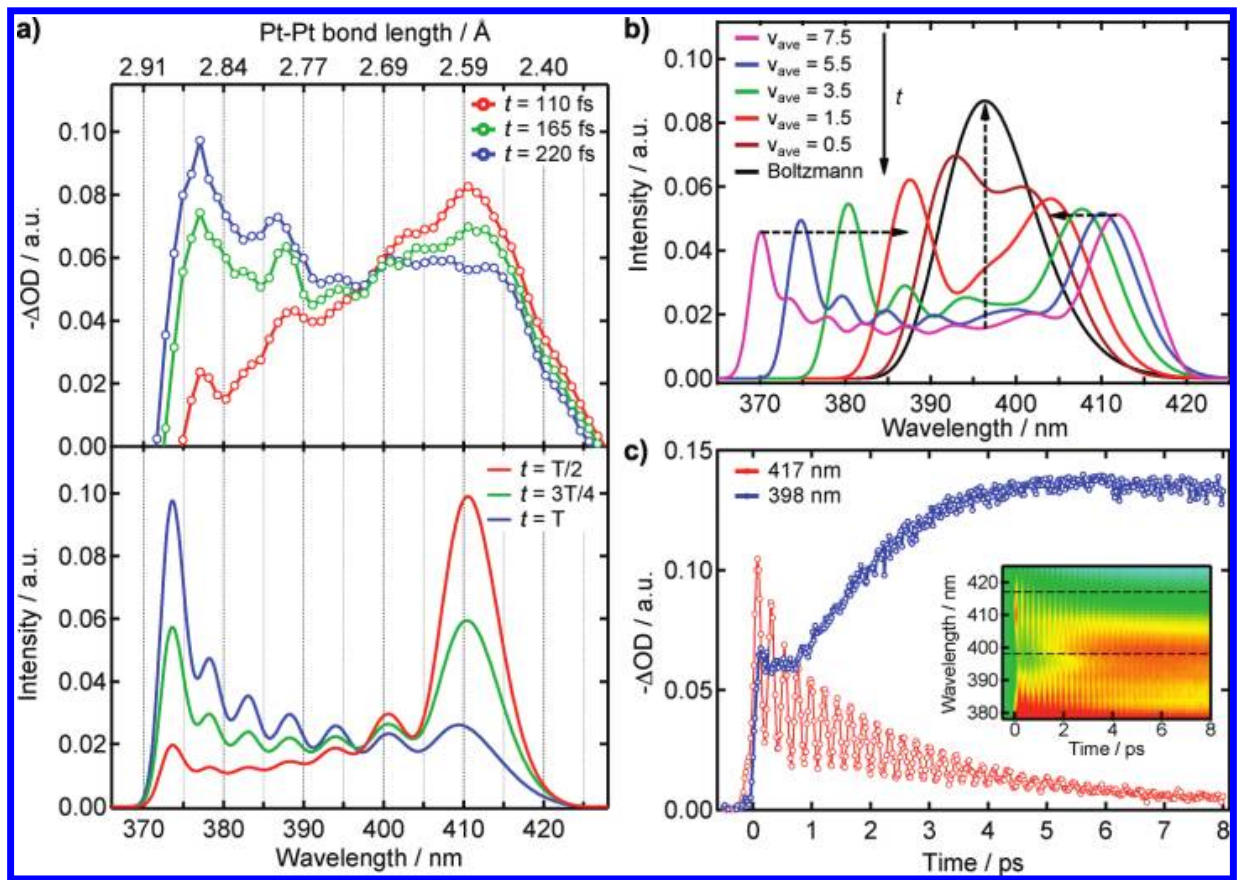

Figure 8. (a) Top: experimental SE spectra at different delay times (PtPOP in ethylene glycol, excitation at $370 \mathrm{~nm}$ ). The scaled static absorption spectrum was subtracted to correct for the partially overlapping GSB. This correction is not fully sufficient in the $370-375 \mathrm{~nm}$ region. Bottom: simulated SE spectra (see text and eq S11 in the Supporting Information) for different time delays expressed as a function of the vibrational period $T=220 \mathrm{fs}$ for an average vibrational quantum number of 6 . The vertical grid lines facilitate the observation of the quantum interference fringes. (b) Theoretical SE spectra for times at which the wave packet passes the middle of the potential for different average wave packet energies. The dashed arrows indicate the trends that are also observed experimentally. (c) Experimental time traces at fixed wavelengths (open circles). The inset shows the corresponding cuts through the 2D plot (dashed lines).

pronounced dependence on the solvent following a similar decreasing trend: ethylene glycol $>$ ethanol $>$ water $>$ DMF. Especially, the ISC rate changes by almost a factor of 3 between ethylene glycol and DMF. For all solvents, coherence decay occurs on the same time scale as vibrational cooling.

2.5. Singlet-State Vibrational Wave Packet Dynamics. Cuts at fixed time delays of the multicolour SE signal provide snapshots of the spectral distribution of the wave packet, as shown in Figure 8a. This is, to our knowledge, the first example where the distribution of the wave packet is spectrally visualized between the turning points of the potential on which it evolves. Given that the emission wavelengths are directly related to the difference potentials at specific internuclear distances, the upper axis in Figure $8 \mathrm{a}$ is obtained by mapping the energy difference between the displaced ${ }^{1} \mathrm{~A}_{2 \mathrm{u}}$ and ${ }^{1} \mathrm{~A}_{1 \mathrm{~g}}$ potential curves (i.e., the emission energy) on the $\mathrm{Pt}-\mathrm{Pt}$ bond length axis. In fact, the SE intensity is directly related to the wave packet probability density via the Franck-Condon factors connecting the ground and excited states (see eq S10 in the Supporting Information). By spectrally resolving the SE spectrum, we thus mapped out the spatial distribution of the wave packet along the full $\mathrm{Pt}-\mathrm{Pt}$ distortion coordinate ranging from 2.91 (ground-state $\mathrm{Pt}-\mathrm{Pt}$ bond length) to $\sim 2.55 \AA$ (at the excited-state far turning point). In Figure $8 \mathrm{a}$, we see that, after half an oscillation, the distribution is peaked at the outer turning point, while at a full oscillation period (220 fs), it is peaked at the inner turning point. At an intermediate time (165 fs) corresponding to $3 / 4$ period, the distribution is nearly uniform over the whole range of distances. This is confirmed by numerical simulations of the characteristic time-resolved shapes of the SE band centered around $400 \mathrm{~nm}$ (Figure 3, details are given in the Supporting Information), where we assume that the excited-state distortion and the vibration involve only the $\mathrm{Pt}-\mathrm{Pt}$ atoms (no ligand contribution), and we used the ground and excited vibrational frequencies derived here. The shape of the fluorescence spectrum and its Stokes shift are then determined by the zero-phonon energy and the difference in equilibrium $\mathrm{Pt}-\mathrm{Pt}$ bond lengths. These two parameters were "fitted" to $25900 \mathrm{~cm}^{-1}(386 \mathrm{~nm})$ and $0.194 \AA$, respectively. The qualitative agreement between experimental and theoretical SE spectra is shown in Figure 8a for different delay times (the scaled static absorption spectrum was subtracted from the experimental SE data to correct for the partially overlapping GSB).

Due to the rather small excitation spectral width (mapping $\sim 3$ vibrational levels) and the difference in vibrational frequency between the ground state and the excited state, the extent of the wave packet undergoes a broadening for $t=T / 4$ and $t=3 T / 4$ and refocusing at the classical turning points for $t=$ $T / 2$ and $t=T$. This behavior of the wave packet is well documented in the literature. ${ }^{21-23}$ The novelty here is the broadband detection, which allows us to spectrally resolve the fine-structured quantum interference fringes corresponding to the nodes of the excited-state vibrational wave function, e.g., occurring at ca. 410, 403, 395, 387, and $377 \mathrm{~nm}$ (see also Figure $3 b){ }^{24}$ The PtPOP molecule thus appears as an ideal harmonic oscillator for the study of environment effects on the vibrational cooling. Figure $8 \mathrm{~b}$ shows the simulation of the SE spectrum at the times when the wave packet passes the middle of the potential for different average vibrational quantum numbers (see

(21) Yeazell, J.; Uzer, T. The physics and chemistry of wave packets; Wiley: New York, 2000

(22) Janszky, J.; Vinogradov, A. Phys. Rev. Lett. 1990, 64, 2771-2774.

(23) Janszky, J.; Adam, P.; Vinogradov, A.; Kobayashi, T. Spectrochim. Acta A 1992, 48, 31-39.

(24) The very blue side of the experimental spectra is obscured by the insufficient GSB subtraction. 
Supporting Information for details). Immediately after excitation at $370 \mathrm{~nm}$, the wave packet has an average energy corresponding to $\sim 7.5$ vibrational quanta (i.e., $v_{\text {ave }}=7.5 \times 150 \mathrm{~cm}^{-1}$ ), relative to the bottom of the potential $(v=0)$.

The process of vibrational cooling is simulated by shifting the wave packet population distribution to lower average vibrational levels. Coherence decay of the wave packet is not included in this simple model; the simulations should therefore only be interpreted qualitatively. From Figure $8 b$ it is seen that the energy dissipation results in a narrowing of the spectrum accompanied by an increase of the intensity in the middle of the band, which matches the behavior observed in Figures 5 $\left(\mathrm{DAS}_{2}\right)$ and $3 \mathrm{~b}$. Finally, the wave packet damps out to a relaxed state given by the Maxwell-Boltzmann distribution, whose spectrum is also shown in Figure 8b. Energy dissipation causes the intensity to decrease monotonically in the wings of the spectrum, while increasing in the middle of the band nonexponentially as a function of the average energy (almost no increase until $v_{\text {ave }}=3.5$, large increase from $v_{\text {ave }}=3.5$ to $\left.v_{\text {ave }}=0.5\right)$. This behavior is also observed experimentally, as shown in Figure 8c. The time trace at $398 \mathrm{~nm}$ exhibits a plateau during the first picosecond before it gradually starts to increase, while the trace at $417 \mathrm{~nm}$ decays monotonically. Due to this nonexponential behavior, it should be kept in mind that the exponential kinetic model for vibrational relaxation employed in the fitting analyses is a simplification of the real dynamics. Interestingly, the deep modulations of the $417 \mathrm{~nm}$ trace in Figure $8 \mathrm{c}$ illustrate the strong localization of the wave packet at the classical turning points of the ${ }^{1} \mathrm{~A}_{2 \mathrm{u}}$ potential.

\section{Discussion}

3.1. Vibrational Population Relaxation in the Singlet State. The above results have shown that there are two kinetics components associated with vibrational energy relaxation in the ${ }^{1} \mathrm{~A}_{2 \mathrm{u}}$ excited state. The first component, $\tau_{1} \simeq 260 \mathrm{fs}$, is slightly longer than the round-trip time of an oscillation in the singlet state. The best agreement between experimental and calculated SE spectra during the first oscillation period after excitation (shown in Figure 8a) was achieved for an average vibrational quantum number of 6 , while excitation mainly populates vibrational levels 7 and 8 at $t=0$. The 260 fs decay component thus involves the dissipation of 1-2 vibrational quanta. This fast population decay component can have different origins. First, it could correspond to the short transient time for which the population relaxation rates of the different levels are not yet fully in phase. ${ }^{25}$ In contrast to the decay of the system as a whole, the population decay of individual levels is generally nonexponential. In ref 26, it is shown that the decay of the highest levels exhibits a fast component comparable to the period of the vibration, similar to the present case. Second, the fast component resembles the case of wave packet dynamics of excited diatomic halogen molecules in rare gas matrices. Schwentner, Apkarian, and co-workers ${ }^{27,28}$ showed that the impulsive bond elongation in the upper (dissociative) part of the potential resulted in a large dissipation of energy (up to 10 quanta of the intramolecular vibration frequency) due to interaction of the halogen atoms with the matrix cage atoms. The coherence was preserved in this process due to the hard

(25) Kohen, D.; Tannor, D. J. Chem. Phys. 1997, 107, 5141-5153.

(26) Jean, J.; Fleming, G. J. Chem. Phvs. 1995, 103, 2092-2101.

(27) Apkarian, V.; Schwentner, N. Chem. Rev. 1999, 99, 1481-1514.

(28) Bihary, Z.; Zadoyan, R.; Karavitis, M.; Apkarian, V. J. Chem. Phys. 2004, 120, 7576-7589. collision of the molecule with the matrix cage in a short interaction time. In the PtPOP case the bond length impulsively shortens. There is less possibility to interact with the solvent for the contracted configuration. However, one could imagine that, during the first contraction, and as a result of the change of electronic structure of the solute, the solvent molecules start to rearrange, leading to an increased solute-solvent interaction with a net transfer of energy to the solvent upon the subsequent elongation, leaving the $\mathrm{Pt}$ atoms vibrating with a reduced amplitude and energy but still in a coherent (wave packet) fashion. Compared to the large elongation event near the dissociation limit for dihalogens, the energy dissipated is much more modest here, because the elongation is not of large amplitude and it stays confined within the "cage" of the bridging ligands.

The second slower kinetics component, $\tau_{2} \simeq 2 \mathrm{ps}$, associated with vibrational cooling, is very sensitive to both excitation wavelength and solvent (Table 1). An estimate of the population lifetime of a single vibronic level can be directly derived from the experimentally determined vibrational cooling time under the assumption that the depopulation rate is independent of the vibrational quantum number $v \cdot{ }^{25} \mathrm{After}$ the aforementioned short transient time, we assume that the depopulation rate is constant for all subsequent vibronic levels until the population reaches the bottom of the potential. The population lifetime is derived by dividing the cooling time $\tau_{2} \simeq 2 \mathrm{ps}$ (corresponding to the time in which the energy is dissipated to $1 / e=36 \%$ of the initial value, i.e., from $v \simeq 6$ to $v \simeq 2$ ) by the number of vibrational quanta that are dissipated, i.e., $\Delta v \simeq 4$. This results in the population lifetime of $\sim 500 \mathrm{fs}$ for a single vibronic level, which raises the question of how the energy is dissipated, and to where?

For large molecules, one generally distinguishes two main channels of vibrational energy dissipation, which usually follow each other in sequence: (1) intramolecular vibrational energy redistribution (IVR) with transfer of energy from the FC-active mode to "dark" vibrational modes of lower energy reaching quasi-thermal equilibrium, ${ }^{29}$ and (2) external vibrational relaxation (EVR), during which the excess vibrational energy is dissipated into the solvent. ${ }^{30}$ In organic molecules of appreciable size ( $>30$ atoms), IVR is typically very fast $(<100 \mathrm{fs})$ due to the large density of vibrational states and high-frequency modes that exhibit anharmonicity already for low vibrational quantum numbers. ${ }^{31-33}$ In the case of PtPOP, however, no vibrational modes of lower energy than the FC-active $\mathrm{Pt}-\mathrm{Pt}$ stretch mode $\left(150 \mathrm{~cm}^{-1}\right)$ have been reported. ${ }^{34,35}$ A $40 \mathrm{~cm}^{-1}$ sideband was superimposed on the $\mathrm{Pt}-\mathrm{Pt}$ vibronic progression in lowtemperature single-crystal spectroscopic studies, but the assignment of this feature as being due to an intramolecular FC-active mode or a phonon mode of the crystal lattice was ambiguous. ${ }^{18,36}$ Given the large mass of the Pt atoms and the fact that the $\mathrm{Pt}-$ ligand coordination bonds are the weakest in the molecule,

(29) Zewail, A. Ber. Bunsen-Ges. Phvs. Chem. 1985, 89, 264-270.

(30) Owrutsky, J.; Raftery, D.; Hochstrasser, R. Annu. Rev. Phys. Chem. 1994, 45, 519-555.

(31) Gawelda, W.; Cannizzo, A.; Pham, V.; van Mourik, F.; Bressler, C.; Chergui, M. J. Am. Chem. Soc. 2007, 129, 8199-8206.

(32) Cannizzo, A.; van Mourik, F.; Gawelda, W.; Zgrablic, G.; Bressler, C.; Chergui, M. Angew. Chem., Int. Ed. 2006, 45, 3174-3176.

(33) Mokhtari, A.; Chesnoy, J.; Laubereau, A. Chem. Phys. Lett. 1989, 155, 593-598.

(34) Stein, P.; Dickson, M.; Roundhill, D. J. Am. Chem. Soc. 1983, 105, 3489-3494.

(35) Gellene, G.; Roundhill, D. J. Phys. Chem. A 2002, 106, 7617-7620.

(36) Bär, L.; Gliemann, G. Chem. Phys. Lett. 1984, 108, 14. 
we can confidently say that the $\mathrm{Pt}-\mathrm{Pt}$ stretch is indeed the lowest-frequency mode in PtPOP. IVR is thus excluded, and consequently, vibrational energy dissipation mainly occurs directly to the solvent.

One can imagine two possible types of solute-solvent interactions mediating the energy flow: (1) interaction between the solvent and the $\mathrm{Pt}$ atoms along the open axial coordination sites, which should be enhanced in the ${ }^{1,3} \mathrm{~A}_{2 \mathrm{u}}\left(\mathrm{d} \sigma^{*}-\mathrm{p} \sigma\right)$ excited states due to their radical character, and (2) interaction of the negatively charged ligand $\mathrm{P}-\mathrm{O}-\mathrm{H} / \mathrm{P}-\mathrm{O}$ groups with polar moieties (e.g., $\mathrm{O}-\mathrm{H}$ groups) of the solvent molecules. For the above reasons, the first mechanism, involving a direct $\mathrm{Pt}-$ solvent interaction, is expected to play a major role in the vibrational relaxation. Indeed, the $\mathrm{Pt}-\mathrm{Pt}$ stretch energy of $150 \mathrm{~cm}^{-1}$ lies in the spectral region dominated by translational and librational modes of the solvent (and solute) molecules, ${ }^{37}$ thus providing resonance modes in the solvent. However, in addition to providing resonance modes, a mechanism is necessary to couple the molecular oscillator to the bath of solvent modes.

The vibrational decay rates (Table 1) can be divided into two groups: ethanol and ethylene glycol with $\tau_{2}>2 \mathrm{ps}$, and water and DMF with $\tau_{2}<1.5 \mathrm{ps}$. The question is what microscopic mechanism lies behind this solvent effect; i.e., what determines the ability of the solvent to accept the vibrational energy from the excited $\mathrm{Pt}-\mathrm{Pt}$ mode? At this stage, in the absence of a detailed microscopic model, we can relate the observed trend to specific solvent properties. One such property is the solvent polarity, which follows the decreasing trend in vibrational relaxation rates: water $>$ DMF $>$ ethylene glycol $>$ ethanol. In addition, the ability of the solvent to interact with the Pt atoms could play a role in energy transfer. Indeed, the ${ }^{3} \mathrm{~A}_{2 u}$ state of PtPOP has been shown to abstract hydrogen atoms from $\mathrm{H}$-atom donors, ${ }^{1}$ e.g., in the photocatalytic conversion of isopropanol into acetone. One can then imagine an interaction of the hydrogen atoms in the solvent $(\mathrm{O}-\mathrm{H}$ in water, ethanol, and ethylene glycol; $\mathrm{C}-\mathrm{H}$ in $\mathrm{DMF}$ ) with the $\mathrm{Pt}$ atoms. In this respect, water and DMF might be better candidates than alcohols, in that they exhibit a larger number of hydrogen atoms that can participate in the $\mathrm{Pt}-\mathrm{H}$ interaction (the methyl hydrogens in DMF have been shown to be better donors than the formyl one ${ }^{38}$ ). Last, the bulkiness of the solvent molecules and the possibility to interact with the polar ligand phosphite groups can play a role in the strength of the coupling. It should be noted that the nature of the solute-solvent interaction is likely to be a transient rather than a static solvent coordination. The latter would lead to a pronounced solvatochromic shift of the steady-state absorption and emission spectra which is not observed experimentally. ${ }^{10}$ The differences in the vibrational cooling rate as a function of the excitation wavelength are attributed to the different amounts of excess vibrational energy that have to be dissipated to the solvent. Because the $\mathrm{Pt}$-solvent interaction can only occur along the Pt-Pt axis (at maximum two solvent molecules coordinate at a certain time), a saturation behavior might arise for large vibrational energies, leading to an increased relaxation time for higher excitation energies, in complete accordance with the present results. This saturation effect of vibrational cooling emphasizes that the solvent does not just act as an infinite sink for excess energy, but it actually

(37) Giraud, G.; Wynne, K. J. Chem. Phys. 2003, 119, 11753-11764.

(38) Lei, Y.; Li, H.; Pan, H.; Han, S. J. Phvs. Chem. A 2003, 107, 15741583. interacts with the solute in a specific way, such that the energy can be funneled only via a limited number of solute-solvent modes.

In summary, we conclude that the main vibrational energy dissipation channel involves direct solute-solvent interaction along the axial coordination sites of the Pt atoms, possibly via $\mathrm{Pt}-\mathrm{H}$ interaction. Although the ligand phosphite groups do not directly funnel vibrational energy from the $\mathrm{Pt}-\mathrm{Pt}$ mode to the solvent (by means of IVR), they may not just be spectators in this picture. The $\mathrm{P}-\mathrm{O} / \mathrm{P}-\mathrm{O}-\mathrm{H}$ groups should play a distinct role in the formation of the hydrogen-bonded solute-solvent network and thus the overall intermolecular interaction.

3.2. Vibrational Wave Packet Dynamics. Remarkable in the process of vibrational relaxation in PtPOP is the exceptional harmonicity of the coherent $\mathrm{Pt}-\mathrm{Pt}$ oscillation. Even for the highest excitation energies, the wave packet oscillation frequency hardly deviates from the low-energy ones, pointing to a harmonic ${ }^{1} \mathrm{~A}_{2 u}$ potential energy surface over a range of more than $2000 \mathrm{~cm}^{-1}$ (ca. 14 vibrational levels). In contrast to common electronic excitations that populate empty antibonding molecular orbitals, often leading to pronounced anharmonicity in the excited-state potential, the $\mathrm{A}_{1 \mathrm{~g}} \rightarrow \mathrm{A}_{2 \mathrm{u}}\left(\mathrm{d} \sigma^{*} \rightarrow \mathrm{p} \sigma\right)$ excitation in bimetallic $\mathrm{d}^{8}-\mathrm{d}^{8}$ complexes involves the formation of a new metal-metal bond in the excited state and thus a steeper vibrational potential compared to the ground state. Despite the rather large $\mathrm{Pt}-\mathrm{Pt}$ contraction of $0.2-0.3 \AA$, the rigid, constraining $\mathrm{P}-\mathrm{O}-\mathrm{P}$ bridging ligands in $\mathrm{PtPOP}$ provide a force constant that is independent of the $\mathrm{Pt}-\mathrm{Pt}$ displacement, giving rise to the highly harmonic vibrational dynamics. This is in contrast to the flexible bridging ligands in the bimetallic complex $\mathrm{Rh}_{2}$ (dimen) ${ }_{4}{ }^{2+}$ (dimen $=1,8$-diisocyanomenthane), for which the excited-state potential surface has been proposed to be extremely anharmonic due to significant torsional ligand deformations. ${ }^{9}$

The coherence persists for a remarkable long time of $\tau_{\mathrm{C}} \simeq 2 \mathrm{ps,}$ much longer than the depopulation time of $\sim 500$ fs of the individual levels. This indicates that the mechanism for depopulation decay in PtPOP does not conform to the optical Bloch picture; ${ }^{26}$ on the microscopic level, energy dissipation to the solvent occurs such that the coherence is preserved to a large extent. We believe that this behavior is due to the exceptional harmonicity of the $\mathrm{Pt}-\mathrm{Pt}$ oscillator, and the fact that it is not entirely exposed to the surroundings, so that solute-solvent interaction can only occur along specific coordinates, most probably the $\mathrm{Pt}-\mathrm{Pt}$ one. The harmonicity guarantees frequency matching between the vibronic levels, resulting in very efficient coherence transfer between vibronic levels. ${ }^{26}$ The cage-like structure of the molecule protects the oscillator from the stochastically fluctuating solvent, also reducing pure dephasing. This behavior shows close resemblance to the vibrational relaxation of dihalogens in rare gas matrices. ${ }^{39,40}$ In those cases, transfer of coherence by collisional interaction of the oscillator with the matrix also provided an unusual relation between population and coherence decay times, with a coherence decay time much longer than the population relaxation time of the initially excited levels. ${ }^{40}$

It should be noted that the wave packet simulations performed in this study showed the best correspondence with the experiment for a $\mathrm{Pt}-\mathrm{Pt}$ contraction of $\sim 0.2 \AA$. This is in satisfying agreement with previous (low-temperature) optical measure-

(39) Guehr, M.; Bargheer, M.; Fushitani, M.; Kiljunen, T.; Schwentner, N. Phys. Chem. Chem. Phys. 2007, 9, 779-801.

(40) Guhr, M.; Schwentner, N. J. Chem. Phys. 2005, 123, 244506. 
ments $(0.175-0.225 \AA),{ }^{8,18,14,41}$ but it is slightly shorter than values for the contraction obtained from time-resolved X-ray crystallography $\left(0.28(9) \AA^{42}\right.$ and $0.23-0.28 \AA^{43-45}$ ), X-ray scattering $\left(0.24(6) \AA^{7}\right)$, and X-ray absorption spectroscopy $\left(0.31(5) \AA^{6,17}\right)$. Although the uncertainties of all derived values are rather large, there is a systematic discrepancy between the values derived by optical and X-ray methods, while the inclusion of small additional distortions, e.g., along Pt-ligand, could change the derived value for the $\mathrm{Pt}-\mathrm{Pt}$ contraction in the optical analysis. In the above discussion we argued that a $\mathrm{Pt}-$ solvent interaction is likely to occur in the excited state. The X-ray diffraction measurements were done with crystalline samples; therefore, care should be taken when comparing with the solution work. The X-ray absorption measurements were performed in solution, but solvent molecules close to the axial Pt coordination sites were not included in the modeling of the signal. For the solution-phase X-ray scattering measurements, the static solute-solvent cross-terms were included in the signal modeling, but their photoinduced changes, e.g., an increased $\mathrm{Pt}-$ solvent interaction in the excited state, are not taken into account.

3.3. Mechanism of Intersystem Crossing. The ${ }^{1} \mathrm{~A}_{2 \mathrm{u}}$ absorption of PtPOP has a molar extinction coefficient of $33500 \mathrm{M}^{-1} \mathrm{~cm}^{-1}$. In contrast, natural and synthetic organic pigments exhibit absorption bands with extinction coefficients that are often in excess of $200000 \mathrm{M}^{-1} \mathrm{~cm}^{-1}$, corresponding to fully allowed dipole transitions. ${ }^{46}$ One of the reason for this lower singlet absorption lies in the very large spin-orbit $(\mathrm{SO})$ coupling constant of $5 \mathrm{~d}\left(\mathrm{Pt}^{\mathrm{II}}\right)$ electrons $\left(4000 \mathrm{~cm}^{-1}\right),{ }^{47}$ which mixes singlet and triplet states, also leading to a weak absorption for the latter. The same can be found in $\mathrm{Fe}-$ and $\mathrm{Ru}$-trisbipyridine complexes. ${ }^{31,32}$ In spite of this large SO coupling, the ISC rate in PtPOP is rather slow (tens of picoseconds), while for other molecules such as $\mathrm{Fe}, \mathrm{Ru}$, Os and Re polypyridine complexes, ${ }^{31,32,48,49} \mathrm{Cr}$ acetylacetones, ${ }^{49}$ and $\mathrm{Fe}$ porphyrins ${ }^{50}$ the ISC times are $<200$ fs typically, even though the metal atoms in these complexes have smaller or comparable SO coupling constants. On the other hand, ISC in mono-Pt(II) acetylides has been shown to occur on ultrafast $(<100 \mathrm{fs})$ time scales. ${ }^{51}$ The above indicates that the ISC rate in metal complexes is not just governed by the SO coupling strengths of the atoms. This was striking in the case of halogenated $\mathrm{Re}$-carbonyl complexes, for which the ISC rate was found to decrease in the sequence $\mathrm{Cl}-\mathrm{Br}-\mathrm{I} .{ }^{48}$ Instead, other

(41) Leung, K.; Phillips, D.; Che, C.; Miskowski, V. J. Raman Spectrosc. 1999, 30, 987-993.

(42) Kim, C.; Pillet, S.; Wu, G.; Fullagar, W.; Coppens, P. Acta Crvstallogr. A 2002, A58, 133-137.

(43) Yasuda, N.; Uekusa, H.; Ohashi, Y. Bull. Chem. Soc. Jpn. 2004, 77, 933-944.

(44) Ozawa, Y.; Terashima, M.; Mitsumi, M.; Toriumi, K.; Yasuda, N.; Uekusa, H.; Ohashi, Y. Chem. Lett. 2003, 32, 62-63.

(45) Yasuda, N.; Kanazawa, M.; Uekusa, H.; Ohashi, Y. Chem. Lett. 2002, 31, 1132-1133.

(46) Hasselman, G. M.; Watson, D. F.; Stromberg, J. R.; Bocian, D. F.; Holten, D.; Lindsey, J. S.; Meyer, G. J. J. Phvs. Chem. B 2006, 110, 25430-25440.

(47) Piepho, S.; Schatz, P.; McCaffer, A. J. Am. Chem. Soc. 1969, 91, 5994.

(48) Cannizzo, A.; Blanco-Rodriguez, A. M.; Nahhas, A. E.; Sebera, J.; Zalis, S.; Vlcek, A.; Chergui, M. J. Am. Chem. Soc. 2008, 130, 89678974.

(49) Juban, E.; Smeigh, A.; Monat, J.; McCusker, J. Coord. Chem. Rev. 2006, 250, 1783-1791.

(50) Harvey, J. Faraday Discuss. 2004, 127, 165-177.

(51) Ramakrishna, G.; Goodson, T.; Rogers-Haley, J.; Cooper, T.; McLean, D.; Urbas, A. J. Phvs. Chem. C 2009, 113, 1060-1066. factors (structure, energetics, symmetry, etc.) contribute to tuning the ISC rate in transition metal complexes.

In the case of dimeric $\mathrm{d}^{8}-\mathrm{d}^{8}$ complexes, the metal-metal interaction largely stabilizes the ${ }^{1,3} \mathrm{~A}_{2 \mathrm{u}}\left(\mathrm{d} \sigma^{*} \rightarrow \mathrm{p} \sigma\right)$ states compared to the corresponding $\mathrm{d}_{z} \rightarrow \mathrm{p}_{z}$ states in monomeric complexes. As a consequence, the ${ }^{1,3} \mathrm{~A}_{2 \mathrm{u}}$ states in PtPOP are the lowest-lying singlet and triplet states. Furthermore, there is a large energy gap between the ${ }^{1,3} \mathrm{~A}_{2 \mathrm{u}}$ states and higher-lying singlet and triplet states. This energetic isolation of the ${ }^{1,3} \mathrm{~A}_{2 u}$ states makes them electronically (except for the spin flip) and geometrically almost identical, which is corroborated by the similar shapes of their steady-state absorption and emission spectra at low temperature ${ }^{10}$ and, as shown here, the very similar ESA spectra (see Figure 5). The Franck-Condon (FC) overlap factors between the singlet- and triplet-state potential curves are therefore very small, and a direct spin-orbit interaction between ${ }^{1} \mathrm{~A}_{2 \mathrm{u}}$ and ${ }^{3} \mathrm{~A}_{2 \mathrm{u}}$ is symmetry forbidden (in $D_{4 h}$ ). Furthermore, they are separated by a large energy gap. This is different from other metal complexes with ultrafast ISC rates, which often exhibit close-lying singlet and triplet states. ${ }^{31,48}$ The question remains, however, what is in fact the mechanism of ISC?

In answering this question, we have to take into account the following experimental findings: (a) The overall dynamics of our broadband TA spectra can be satisfactorily fitted with a twostate model involving direct interconversion from the ${ }^{1} \mathrm{~A}_{2 \mathrm{u}}$ state to the ${ }^{3} A_{2 u}$ state (Figures 5 and 7). There is no evidence for the population of a third excited state. (b) The wave packet dynamics are extremely harmonic with a rather long coherence decay time, pointing to a largely unperturbed singlet-state potential (Figure 6). Therefore, coupling does not occur along the $\mathrm{Pt}-\mathrm{Pt}$ coordinate, and we can exclude strong coupling between the $\mathrm{Pt}-\mathrm{Pt}$ coordinate and other intramolecular modes. (c) The ISC rate is strongly solvent-dependent (Table 1). (d) The ISC rate follows an Arrhenius-type dependence, as shown by Midler and Brunschwig, ${ }^{13}$ who suggested that ISC takes place via two channels: temperature-independent and temperaturedependent ones, which are the dominating channels at low and high temperatures, respectively. At room temperature the latter is $\sim 18$ faster than the temperature-independent channel. Because our measurements were performed at room temperature, in the following we discuss only this regime of ISC.

The above results can be reconciled within various models of ISC listed below:

(1) ISC takes place through a mechanism of spin-vibronic coupling according to $\left\langle\phi_{a}\left|h_{s o}\right| \phi_{b}\right\rangle\left\langle\phi_{b}\left|\partial / \partial Q_{i}\right| \phi_{c}\right\rangle$, where $\phi_{a}$ corresponds to the ${ }^{1} \mathrm{~A}_{2 \mathrm{u}}$ state which $\mathrm{SO}$ couples with the perturbing state $\phi_{b}{ }^{52}$ The latter in turn vibronically couples with state $\phi_{c}$, corresponding to the ${ }^{3} \mathrm{~A}_{2 \mathrm{u}}$ state, through a vibrational mode $Q_{i}$. As the perturbing state that lies relatively close in energy to the ${ }^{1} \mathrm{~A}_{2 \mathrm{u}}$ state, we propose the ${ }^{3} \mathrm{E}_{\mathrm{u}}$ state resulting from ligandto-metal $\mathrm{p}_{x, y}(\mathrm{P}) \rightarrow 6 \mathrm{p}_{z}(\mathrm{Pt})$ excitation. ${ }^{5}$ This is in contrast to the metal-centered ${ }^{3} \mathrm{E}_{\mathrm{u}}\left(\mathrm{d}_{x z, y z} \rightarrow \mathrm{p}_{z}\right)$ state proposed in ref 15 , which lies much higher in energy. Modes of $e_{g}$ symmetry can vibronically couple the ${ }^{3} \mathrm{~A}_{2 \mathrm{u}}$ and ${ }^{3} \mathrm{E}_{\mathrm{u}}$ states. Within this picture, the solvent effect could be explained by (i) a solvent-dependent stabilization energy of the charge-transfer ${ }^{3} \mathrm{E}_{\mathrm{u}}$ state that is distorted along the $\mathrm{Pt}$-ligand coordination bonds (the ${ }^{1} \mathrm{~A}_{2 \mathrm{u}}-{ }^{3} \mathrm{E}_{\mathrm{u}}$ energy gap enters in the denominator of the rate expression; such an effect is not expected for the "intra-Pt" ${ }^{3} \mathrm{E}_{\mathrm{u}}\left(\mathrm{d}_{x z, y z} \rightarrow \mathrm{p}_{z}\right)$ state) or (ii) the ability of the solvent to bridge the energy gap

(52) Lin, S. J. Chem. Phvs. 1966, 44, 3759. 
between the vibronic states (ensuring energy conservation during the deactivation). The temperature dependence could enter via the Boltzmann-averaged FC factors; i.e., ISC becomes faster the more vibrational levels are populated thermally.

(2) ISC is induced by symmetry lowering in the excited state. Low-temperature phosphorescence measurements have indicated $D_{4 h}$ molecular symmetry in the ${ }^{3} \mathrm{~A}_{2 \mathrm{u}}$ state, ${ }^{14}$ for which direct SO interaction between ${ }^{1} \mathrm{~A}_{2 u}$ and ${ }^{3} \mathrm{~A}_{2 \mathrm{u}}$ states is symmetry forbidden. A transiently induced symmetry lowering, e.g., toward $D_{4}$ symmetry, would make such an interaction allowed. The symmetry breaking could be induced by the solvent interacting with the radical $\mathrm{Pt} \mathrm{d} \sigma^{*}$ orbital at the open axial coordination sites (the solvent-induced change in angular momentum is accompanied by a spin flip of the electron residing in the $\mathrm{d} \sigma^{*}$ orbital). Alternatively, the $D_{4 h}$ structure could be in equilibrium with a conformation of lower symmetry lying slightly higher in energy, for which ISC is enhanced. This model would rationalize both the experimentally observed solvent and temperature effects, the former through the solvent-dependent stabilization energies of the lower-symmetry conformation, the latter via a thermal activation barrier between the two conformations. Indeed, isomeric structures of different symmetry and energy have been calculated for PtPOP. ${ }^{35}$ The isomeric conformers differ in the hydrogen-bonding motif (eclipsed vs staggered) and $\mathrm{Pt}-\mathrm{P}-\mathrm{O}-\mathrm{P}$ dihedral angle; the highest-symmetry (eclipsed) structure lies $0.8 \mathrm{kcal} / \mathrm{mol}$ lower in energy than the lower-symmetry (staggered) one.

(3) ISC occurs via the thermal population of a higher-lying triplet state. This mechanism has been proposed by Midler and Brunschwig. ${ }^{13}$ They invoke a ligand-field ${ }^{3} \mathrm{~B}_{2 \mathrm{u}}\left(\mathrm{d} \sigma^{*} \rightarrow \mathrm{d}_{x^{2}-y^{2}}\right)$ state that vibronically couples with the ${ }^{1} \mathrm{~A}_{2 \mathrm{u}}$ state along the $\mathrm{Pt}$-ligand coordinate. The solvent effect on the ISC rate is assigned to the different stabilization energies of the ${ }^{3} \mathrm{~B}_{2 u}$ state; decreasing solvent polarity lowers the activation barrier by stabilizing the ${ }^{3} \mathrm{~B}_{2 u}$ state, resulting in an increased ISC rate. This is exactly the opposite of what is observed in the present study. In addition, the population of the ${ }^{3} B_{2 u}$ state is spin forbidden. For this model, we would therefore propose the ${ }^{3} \mathrm{E}_{\mathrm{u}}$ $\left(\mathrm{p}_{x, y}(\mathrm{P}) \rightarrow 6 \mathrm{p}_{z}(\mathrm{Pt})\right)$ state as a possible thermally activated and SOallowed pathway. It is noted that this model is not in contradiction with the fact that we found a good fit of the data to a twostate relaxation model. If the ${ }^{3} E_{u}$ state relaxes to the ${ }^{3} A_{2 u}$ state on the time scale of hundreds of femtoseconds (or faster), the transient population of this state would be too low to be experimentally detected. This model implies a strong perturbation of the potential along the distortion coordinate (depending on the nature of the ${ }^{3} E_{u}$ state). Such a perturbation is likely to influence the vibrational dynamics along the $\mathrm{Pt}-\mathrm{Pt}$ coordinate via mode coupling and anharmonicity (manifested in a frequency chirp). This is clearly not the case here; the highly harmonic wave packet dynamics is a result of a largely unperturbed potential energy surface.

In summary, we conclude that the first two models or a combination of both are the most likely mechanisms of ISC in PtPOP. The occurrence of non-totally symmetric modes of $e_{g}$ symmetry could be confirmed by ultrafast X-ray absorption spectroscopy studies $^{53}$ detecting the transiently distorted geometries.

(53) Bressler, C.; Milne, C. J.; Pham, V.-T.; Nahhas, A. E.; van der Veen, R. M.; Gawelda, W.; Johnson, S.; Beaud, P.; Grolimund, D.; Kaiser, M.; Borca, C. N.; Ingold, G.; Abela, R.; Chergui, M. Science 2009, 323, 489-492.

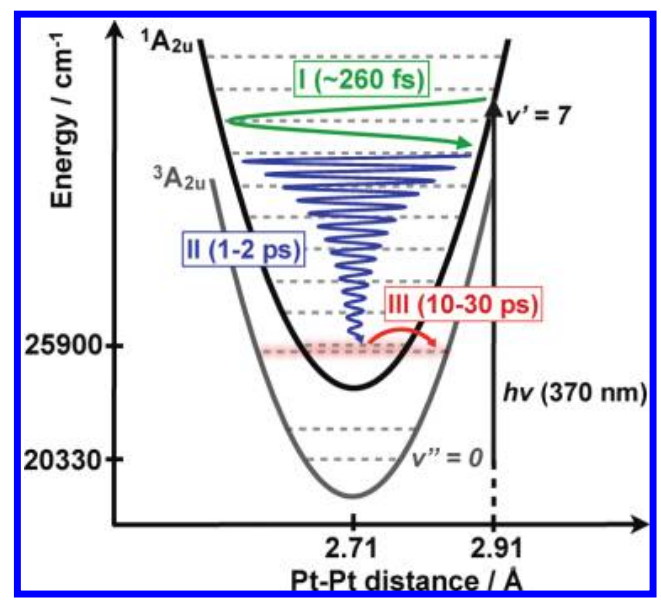

Figure 9. Potential energy level diagram summarizing the relative energies and the characteristic time scales of the various relaxation processes for PtPOP. (I) Impulsive vibrational cooling during the first $\mathrm{Pt}-\mathrm{Pt}$ oscillation dissipating about two vibrational quanta. (II) Solvent-dependent vibrational cooling at a rate of $\sim 35 \mathrm{~cm}^{-1} / 100$ fs and concomitant coherence decay. (III) Intersystem crossing (solvent-dependent).

\section{Conclusions}

Femtosecond fluorescence up-conversion and femtosecond broadband TA measurements, combined with SVD and global fit analysis techniques, have allowed us to identify wave packet dynamics in the ${ }^{1} \mathrm{~A}_{2 \mathrm{u}}$ state potential with a period of $224 \mathrm{fs}$, coherence decay and concomitant vibrational cooling in $1-2 \mathrm{ps}$ in the ${ }^{1} \mathrm{~A}_{2 \mathrm{u}}$ state potential, and ISC on the time scale of 10-30 ps. The latter two processes show a pronounced solvent dependence. The complete scenario of the excited-state dynamics and relaxation is schematically presented in Figure 9. The extreme harmonicity of the $\mathrm{Pt}-\mathrm{Pt}$ wave packet dynamics indicates an unperturbed ${ }^{1} \mathrm{~A}_{2 u}$ state potential energy surface.

We find that vibrational relaxation involves energy dissipation to the solvent, likely involving $\mathrm{Pt}-\mathrm{H}$ interactions along the open axial coordination sites. Due to the absence of vibrational modes with lower frequency than the Pt-Pt stretch, IVR is excluded in PtPOP. The highly harmonic wave packet dynamics independent of the excitation wavelength point to an exceptionally deep potential along the $\mathrm{Pt}-\mathrm{Pt}$ coordinate. The vibrational depopulation time is as short as $\sim 500 \mathrm{fs}$; nevertheless, the vibrations remain coherent during the vibrational cooling time of $\sim 2$ ps. We thus observe the fascinating process in which depopulation occurs without distorting the phases of the coherences; the standard relationship between phase and energy relaxation derived from the optical Bloch equations cannot be used in this case. Coherence transfer between the vibronic levels in the harmonic ${ }^{1} \mathrm{~A}_{2 u}$ potential is thus very efficient. The high harmonicity and the consequently long coherence decay time are identified as being due to the constraining $\mathrm{P}-\mathrm{O}-\mathrm{P}$ bridging ligands that protect the $\mathrm{Pt}-\mathrm{Pt}$ oscillator from random solvent fluctuations.

The low ISC rate compared to other metal complexes with smaller SO coupling constants is attributed to the facts that (i) direct SO coupling between two relevant states (namely ${ }^{1} \mathrm{~A}_{2 \mathrm{u}}$ and ${ }^{3} \mathrm{~A}_{2 \mathrm{u}}$ ) is symmetry forbidden; (ii) the singlet and triplet states lie far apart from each other and from the higher lying excited states of the molecule; and (iii) the singlet and triplet potential curves are parallel, which implies very low Franck-Condon overlaps of the $v=0$ level of the ${ }^{1} \mathrm{~A}_{2 u}$ state with the resonant vibrational levels of the ${ }^{3} \mathrm{~A}_{2 \mathrm{u}}$ state. Other metal-based systems of $\operatorname{Pt}(0)$ and $\mathrm{Cu}(\mathrm{I})$ show similar slow (3-15 ps) ISC rates 
between singlet and triplet MLCT states. ${ }^{54-56}$ Just as in the case of PtPOP, the lowest singlet and triplet potential curves in these complexes are parallel (identical geometry), and direct SO interaction between them is forbidden. DFT calculations have shown that structural distortions in the singlet MLCT state cause large energy splitting between HOMO and HOMO-1, preventing a very fast ISC induced by strong spin-orbit interactions between these orbitals, which are mainly composed of metal d-orbitals. The relatively slow ISC is therefore induced by weak spin-orbit interactions with nearby triplet MLCT states with small d-orbital contribution. In PtPOP, among all low-lying triplet states, SO interaction is allowed only for the ${ }^{3} \mathrm{E}_{u} \mathrm{LMCT}$ state. This interaction is expected to be relatively weak due to the small Pt d-orbital contribution in the underlying molecular orbitals. However, DFT and quantum mechanical SO overlap calculations for the excited-state geometry need to be performed in order to quantify this. In general, we can conclude that the large variability of values of the ISC rate among metal-based molecular complexes can be rationalized by considering the structural and energetic constraints that affect the rate in spite of very large SO coupling constants in all cases. The present study shows that this can be generalized to ISC involving ligandfield and LMCT states.

(54) Siddique, Z.; Yamamoto, Y.; Ohno, T.; Nozaki, K. Inorg. Chem. 2003, 42, 6366-6378

(55) Abedin-Siddique, Z.; Ohno, T.; Nozaki, K. Inorg. Chem. 2004, 43, 663-673.

(56) Shaw, G.; Grant, C.; Shirota, H.; Castner, E. W.; Meyer, G. J.; Chen, L. X. J.Am. Chem. Soc. 2007, 129, 2147-2160.
We believe that the wave packet dynamics observed in PtPOP is a general phenomenon for binuclear $\mathrm{d}^{8}-\mathrm{d}^{8}$ complexes excited to the ${ }^{1} \mathrm{~A}_{2 u}$ state. However, changes in the metal atoms and/or ligands are expected to have a large impact on ultrafast relaxation steps, such as the wave packet oscillation frequency and the rates of vibrational cooling and ISC and the accompanying structural distortions. With the combined approach of femtosecond optical and X-ray techniques ${ }^{53}$ it would be possible to fully map out the electronic and structural changes during the relaxation.

Acknowledgment. This article is dedicated to Prof. N. Schwentner on the occasion of his 65th birthday. The authors thank J. A. Weinstein and J. Best (University of Sheffield) for providing the sample, O. Bräm and C. J. Milne (EPFL) for assistance during the measurements, and P. Coppens (SUNY Buffalo) for useful discussions. This work has been supported by the European collaboration program COST D35 "From Molecules to Molecular Devices" and the European Science Foundation DYNA network "Ultrafast Structural Dynamics in Physics, Chemistry, Biology and Materials Science".

Supporting Information Available: Experimental details, setup description, SVD/GF and GA theoretical details, SVD/ GF and GA results for transient absorption and emission data for different excitation wavelengths and solvents, theoretical details of the wave packet, and stimulated emission simulations. This material is available free of charge via the Internet at http:// pubs.acs.org.

JA106769W 